\title{
Recent Advances in Semiconductor Surface-Emitting Lasers
}

\author{
Krassimir Panajotov, ${ }^{1,2}$ Rainer Michalzik, ${ }^{3}$ and Kent D. Choquette ${ }^{4}$ \\ ${ }^{1}$ Department of Applied Physics and Photonics, Vrije Universiteit Brussel, Pleinlaan 2, 1050 Brussels, Belgium \\ ${ }^{2}$ Georgi Nadjakov Institute of Solid State Physics, Bulgarian Academy of Sciences, 72 Tzarigradsko Chaussee Boulevard, \\ 1784 Sofia, Bulgaria \\ ${ }^{3}$ Institute of Optoelectronics, Ulm University, Albert-Einstein-Allee 45, $89081 \mathrm{Ulm}$, Germany \\ ${ }^{4}$ Department of Electrical and Computer Engineering, University of Illinois, 208 North Wright Street, Urbana, IL 61801, USA
}

Correspondence should be addressed to Krassimir Panajotov, kpanajot@b-phot.org

Received 23 August 2012; Accepted 23 August 2012

Copyright (C) 2012 Krassimir Panajotov et al. This is an open access article distributed under the Creative Commons Attribution License, which permits unrestricted use, distribution, and reproduction in any medium, provided the original work is properly cited.

This special issue 1 of Advances in Optical Technologies contains 12 review papers highlighting the most recent and impressive achievements and exciting new concepts in the field of semiconductor surface-emitting lasers. Hereafter we briefly summarize its content.

Two review articles present advances in the development of semiconductor disk lasers (SDLs). M. Guina et al. from Tampere University of Technology review SDLs producing yellow-orange and mid-IR radiation for high-impact applications in medicine, spectroscopy, or astronomy. They demonstrate dilute nitride (GaInNAs) gain mirror SDLs with more than $11 \mathrm{~W}$ output power in the wavelength range 1180$1200 \mathrm{~nm}$ and subsequent intracavity frequency doubling to generate yellow-orange radiation with power exceeding $7 \mathrm{~W}$. For the 2-3 $\mu \mathrm{m}$ wavelength range, they use GaSb gain mirrors and achieve the shortest pulses of $384 \mathrm{fs}$ obtained from SDLs at $2 \mu \mathrm{m}$. A paper from the group of E. Kapon at the Swiss Federal Institute of Technology in collaboration with the Technical University of Lodz, Poland reviews, the progress in SDLs based on wafer-fused InAlGaAs/InP-AlGaAs/GaAs gain mirrors for telecommunication wavelengths. Continuouswave $(\mathrm{CW})$ output powers of $2.7 \mathrm{~W}$ at $1550 \mathrm{~nm}, 4.8 \mathrm{~W}$ at $1480 \mathrm{~nm}$, and $6.6 \mathrm{~W}$ at $1300 \mathrm{~nm}$ have been demonstrated, as well as second-harmonic emission at $650 \mathrm{~nm}$ wavelength with a record output of $3 \mathrm{~W}$ and Raman fiber lasers with $0.5 \mathrm{~W}$ emission at $1600 \mathrm{~nm}$.

A. Mutig and D. Bimberg from the Technische Universität Berlin, Germany, review the progress on high-speed $980 \mathrm{~nm}$ vertical-cavity surface-emitting lasers (VCSELs) for shortreach optical interconnects, reporting direct modulation rates exceeding $40 \mathrm{Gbit} / \mathrm{s}$ and excellent temperature stability.
The major device concepts leading to these impressive achievements are presented.

A review paper from the group of R. Michalzik at Ulm University, Germany, presents the monolithic integration, fabrication, and electro-optical properties of $\mathrm{AlGaAs} / \mathrm{GaAs}-$ based transceiver (TRx) chips for $850 \mathrm{~nm}$ wavelength optical links. Two design concepts are presented, based on a VCSEL and a monolithically integrated PIN-type photodiode for 62.5 and $50 \mu \mathrm{m}$ core diameter multimode fiber (MMF) or metal-semiconductor-metal photodetector for 100 or $200 \mu \mathrm{m}$ large-area fibers. The feasibility of upgrading standard MMF networks to handle true bi-directional data rates of up to $10 \mathrm{Gbit} / \mathrm{s}$ is demonstrated.

K. Johnson et al. from Vixar company in Plymouth, USA, review the advances in red VCSEL technology for medical and industrial sensing, printing, scanning, and lower cost interconnects based upon plastic optical fiber. Some of the state-of-the-art red VCSEL performance demonstrations include output power of $14 \mathrm{~mW} \mathrm{CW}$ at room temperature, a record maximum temperature of $105^{\circ} \mathrm{C}$ for $\mathrm{CW}$ operation at an emission wavelength of $680 \mathrm{~nm}$, time to $1 \%$ failure at room temperature of approximately 200,000 hours, lifetime in a $50^{\circ} \mathrm{C}, 85 \%$ humidity environment in excess of 3500 hours, digital data rates of $3 \mathrm{Gbit} / \mathrm{s}$, and peak pulsed array powers greater than $100 \mathrm{~mW}$.

K. D. Choquette et al. from the University of Illinois, in collaboration with the Rose-Hulman Institute of Technology, Terre Haute, and the United States Military Academy West Point, all from the USA, and with the National University of Singapore, review the design, fabrication, and performance of photonic crystal VCSELs for enhanced single-transverse 
mode operation. They demonstrated that by control of the refractive index and loss created by the photonic crystal, operation in the Gaussian mode can be ensured, independent of the lasing wavelength.

N. Gerhardt and M. Hofmann from the Ruhr-University Bochum, Germany, review the progress in spin-controlled VCSELs. By means of optical excitation and numerical simulations, they present superior properties compared to conventional devices such as threshold reduction, spin control of the emission, or even much faster dynamics. Possible concepts for room-temperature electrical spin injection without large external magnetic fields are summarized, and the progress on the field of purely electrically pumped spinVCSELs is reviewed.

M. I. Hill and M. I. H. Marell from the Technische Universiteit Eindhoven, The Netherlands, review the advances in surface-emitting metal nanocavity lasers. Two different realized versions of these nanopillar devices, one with a trapped cutoff mode in the pillar, another with a mode that propagates along the pillar, have been demonstrated.

K. Panajotov et al. from the Vrije Universiteit Brussel, Belgium, in collaboration with Supélec in Metz, France, and the Universidad Pública de Navarra in Pamplona, Spain, review the VCSEL nonlinear dynamics induced by optical injection, optical feedback, current modulation, and mutual coupling. For the case of orthogonal optical injection, they demonstrate a new Hopf bifurcation that delimits the injection locking region and a new resonance tongue that is due to first-order transverse mode injection locking. Similarly, the underlying polarization mode competition leads to chaotic-like behavior in case of gain switching and to anticorrelated polarization dynamics in the regimes of lowfrequency fluctuations and self-pulsations induced by optical feedback. Polarization dynamics, however, improves chaos synchronization between unidirectionally coupled VCSELs.

R. Sarzała et al. from the Technical University of Lodz, Poland in collaboration with the Vrije Universiteit Brussel, Belgium, review the progress in self-consistent modeling of optical, electrical, thermal, and carrier transport phenomena taking part in VCSELs. Versatile numerical methods for nitride, arsenide, and phosphide VCSELs emitting light from violet to near-infrared with either gain guiding by a tunnel junction or index guiding by oxide or photonic crystal aperture are presented, focusing on enhancing the singletransverse-mode operation.

V. Bardinal et al. from LAAS-CNRS and Université de Toulouse, France, review the main recent technological approaches to combine micro-optical elements with VCSELs in order to control their output beam and to improve their photonic integration. They detail their integration techniques of self-aligned polymer microlens fabrication for beam collimation and short-distance beam focusing.

Finally, S. Barbay and R. Kuszelewicz from Laboratoire de Photonique et de Nanostructures at CNRS, Marcoussis, in collaboration with J. Tredicce from the Institut Non Linéaire de Nice, both in France, review the advances on the experimental study of cavity solitons (CS) in VCSELs during the past decade. They emphasize on the design and fabrication of electrically or optically pumped broad-area VCSELs used for
CS formation, review different experimental configurations and discuss CS applications for all-optical processing of information and for VCSEL characterization.

\section{Acknowledgments}

It was our great pleasure and honor to work for the success of this special issue. We thank the contributors for their careful writing and report on significantly new results. Many thanks go to all referees for their hard and prompt work. As a result, this special issue provides a relevant snapshot of the most recent advances to the field, and we hope that it will motivate further work on the basis of these findings, concepts, and applications. We thank the editorial staff of Advances in Optical Technologies for their kind attention and their efforts in making this special issue possible.

Krassimir Panajotov
Rainer Michalzik
Kent D. Choquette 

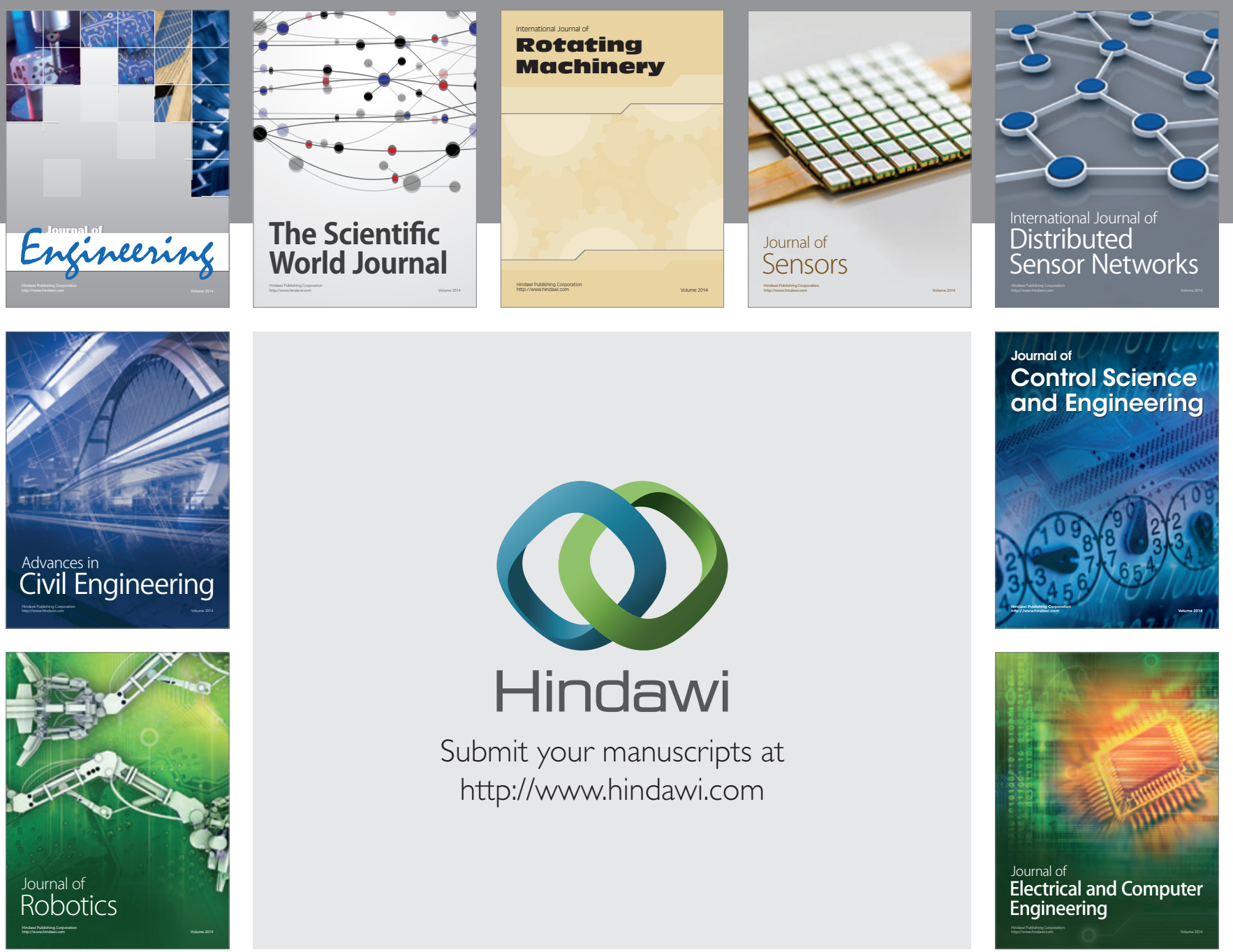

Submit your manuscripts at

http://www.hindawi.com
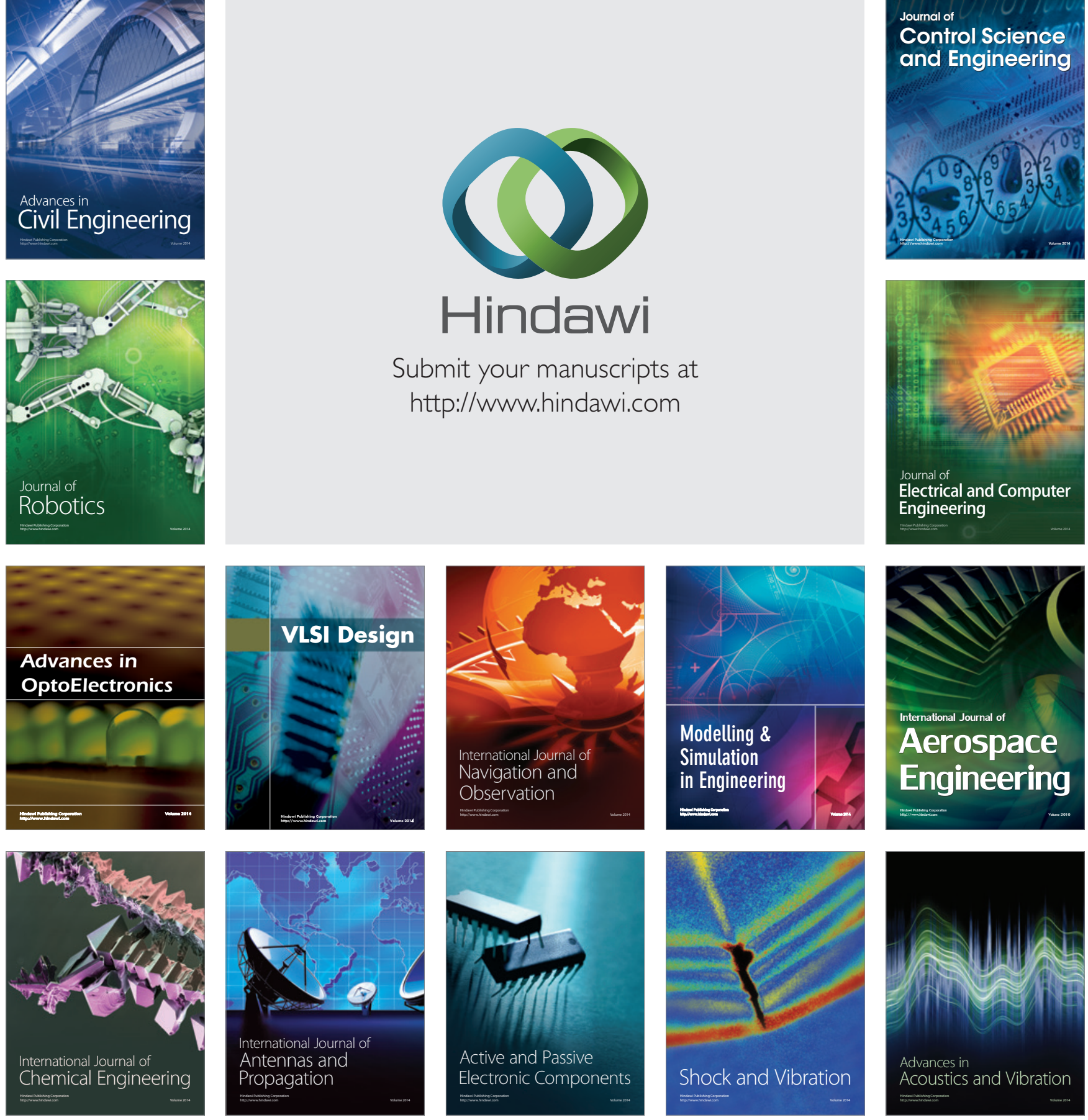